INPLASY

PROTOCOL

To cite: Duan et al. Survival and Neurologic Outcome of Different Time of Collapse to return of Spontaneous Circulation in Cardiac Arrest with Targeted Temperature Management: a Bayesian Network Meta-analysis. Inplasy protocol 202180027. doi:

10.37766/inplasy2021.8.0027

Received: 08 August 2021

Published: 08 August 2021

Corresponding author:

Qingbian Ma

maqingbian@medmail.com.cn

Author Affiliation:

Peking University Third

Hospital.

Support: None.

Review Stage at time of this submission: The review has not yet started.

Conflicts of interest:

None declared.

\section{Survival and Neurologic Outcome of Different Time of Collapse to return of Spontaneous Circulation in Cardiac Arrest with Targeted Temperature Management: a Bayesian Network Meta-analysis}

Duan, J1; Yu, J2; Zhai, Q33 Ma, Q4 .

Review question / Objective: P: patents with cardiac arrest and received targeted temperature management (TTM) or not; I: the different time of collapse to return of spontaneous circulartion (<20min+TTM, <20min, 20-39min+TTM, 20-39min, 40-59min+TTM, 40-59min, $\geq 60 \mathrm{~min}+$ TTM, $\geq 60 \mathrm{~min}$ ); C: same as ablove; 0: 30-day or at discharge survival and good neurologic outcome; S: all type study.

Condition being studied: Trials that met the following criteria were included: a) patients in the trial underwent $C A$ whether IHCA or OHCA; b) valid data of 30-day or at discharge survival neurologic outcome could be extracted; c) the time of collapse to ROSC could be extracted and we could divide into groups based on the time of collapse to ROSC; d) if patients receive TTM the temperature should range from $32^{\circ} \mathrm{C}$ to $36^{\circ} \mathrm{C}$.

INPLASY registration number: This protocol was registered with the International Platform of Registered Systematic Review and Meta-Analysis Protocols (INPLASY) on 08 August 2021 and was last updated on 08 August 2021 (registration number INPLASY202180027).

\section{INTRODUCTION}

Review question / Objective: P: patents with cardiac arrest and received targeted temperature management (TTM) or not; I: the different time of collapse to return of spontaneous circulartion (<20min+TTM, $<20 \mathrm{~min}, 20-39 \mathrm{~min}+$ TTM, 20-39min, 40-59min+TTM, 40-59min, $\geq 60 \mathrm{~min}+$ TTM, $\geq 60 \mathrm{~min}$ ); C: same as ablove; 0: 30 -day or at discharge survival and good neurologic outcome; S: all type study. 
Condition being studied: Trials that met the following criteria were included: a) patients in the trial underwent CA whether IHCA or OHCA; b) valid data of 30-day or at discharge survival neurologic outcome could be extracted; c) the time of collapse to ROSC could be extracted and we could divide into groups based on the time of collapse to ROSC; d) if patients receive TTM the temperature should range from $32^{\circ} \mathrm{C}$ to $36^{\circ} \mathrm{C}$.

\section{METHODS}

Search strategy: PubMed: ("Cardiopulmonary Resuscitation"[Mesh] or Resuscitation, Cardiopulmonary or CPR or Cardio-Pulmonary Resuscitation or Cardio Pulmonary Resuscitation or Resuscitation, Cardio-Pulmonary or Code Blue or Mouth-to-Mouth Resuscitation or Mouth to Mouth Resuscitation or Mouthto-Mouth Resuscitations or Resuscitation, Mouth-to-Mouth or Resuscitations, Mouthto-Mouth or Basic Cardiac Life Support or Life Support, Basic Cardiac) and ("Hypothermia, Induced"[Mesh] or Therapeutic Hypothermia or Hypothermia, Therapeutic or Targeted Temperature Management or Targeted Temperature Managements or Induced Hypothermia or Moderate Hypothermia, Induced or Induced Moderate Hypothermia or Induced Moderate Hypothermias or Moderate Hypothermias, Induced or Mild Hypothermia, Induced or Induced Mild Hypothermia or Induced Mild Hypothermias or Mild Hypothermias, Induced) and ("Heart Arrest"[Mesh] or Arrest, Heart or Cardiac Arrest or Arrest, Cardiac or Asystole or Asystoles or Cardiopulmonary Arrest or Arrest, Cardiopulmonary or ((pulseless* or nonperfus*) and (ventricular tachycardia* or ventricular tachyarrhythmia)) or ventricular fibrillation* or Pulseless electrical activity or PEA). Embase: \#1 Cardiopulmonary Resuscitation/exp ; \#2 Resuscitation, Cardiopulmonary; \#3 CPR; \#4 CardioPulmonary Resuscitation; \#5 Code Blue; \#6 Mouth-to-Mouth Resuscitation*; \#7 Mouthto-Mouth; \#8 Basic Cardiac Life Support; \#9 Life Support, Basic Cardiac; \#10 \#1 or \#2 or \#3 or \#4 or \#5 or \#6 or \#7 or \#8 or \#9; \#11 Hypothermia, Induced; \#12 Therapeutic
Hypothermia/exp; \#13 Targeted Temperature Management*; \#14 TTM; \#15 Induced Hypothermia; \#16 Moderate Hypothermia, Induced; \#17 Induced Moderate Hypothermia*; \#18 Induced Mild Hypothermia*; \#19 \#11 or \#12 or \#13 or \#14 or \#15 or \#16 or \#17 or \#18; \#20 Heart Arrest/exp; \#21 Cardiac Arrest; \#22 Asystole*; \#23 Cardiopulmonary Arrest; \#24 Ventricular fibrillation*; \#25 Pulseless electrical activity; \#26 PEA; \#27 \#20 or \#21 or \#22 or \#23 or \#24 or \#25 or \#26; \#28 Pulseless*; \#29 Nonperfus*; \#30 Ventricular tachycardia *; \#31 Ventricular tachyarrhythmia; \#32 \#28 or \#29; \#33 \#30 or; \#31; \#34 \#32 and \#33; \#35 \#27 or \#34; \#36 \#10 and \#19 and \#35. CENTRAL: \#1 MeSH descriptor: [Cardiopulmonary resuscitation] explode all trees; \#2 Resuscitation, Cardiopulmonary; \#3 CPR; \#4 Cardio-Pulmonary Resuscitation; \#5 Code Blue; \#6 Mouth-to-Mouth Resuscitation*; \#7 Mouth-to-Mouth; \#8 Basic Cardiac Life Support; \#9 Life Support, Basic Cardiac; \#10 \#1 or \#2 or \#3 or \#4 or \#5 or \#6 or \#7 or \#8 or \#9; \#11 MeSH descriptor: [Hypothermia, Induced] explode all trees; \#12 Therapeutic Hypothermia; \#13 MeSH descriptor: [Targeted Temperature Management] explode all trees; \#14 TTM; \#15 Induced Hypothermia; \#16 Moderate Hypothermia, Induced; \#17 Induced Moderate Hypothermia*; \#18 Induced Mild Hypothermia*; \#19 \#11 or \#12 or \#13 or \#14 or \#15 or \#16 or \#17 or \#18; \#20 MeSH descriptor: [Heart Arrest] explode all trees; \#21 Cardiac Arrest; \#22 MeSH descriptor: [Asystole] explode all trees; \#23 Asystole*; \#24 Cardiopulmonary Arrest; \#25 MeSH descriptor: [Ventricular fibrillation] explode all trees; \#26 Pulseless electrical activity; \#27 PEA; \#28 \#20 or \#21 or \#22 or \#23 or \#24 or \#25 or \#26 or \#27; \#29 Pulseless*; \#30 Nonperfus*; \#31 MeSH descriptor: [Ventricular tachycardia] explode all trees; \#32 Ventricular tachyarrhythmia; \#33 \#29 or \#30; \#34 \#31 or \#32; \#35 \#33 and \#34; \#36 \#28 or \#35; \#37 \#10 and \#19 and \#36. Web of science: \#1 all=(Cardiopulmonary Resuscitation or Resuscitation, Cardiopulmonary or CPR or CardioPulmonary Resuscitation or Cardio Pulmonary Resuscitation or Resuscitation, 
Cardio-Pulmonary or Code Blue or Mouthto-Mouth Resuscitation or Mouth to Mouth Resuscitation or Mouth-to-Mouth Resuscitations or Resuscitation, Mouth-toMouth or Resuscitations, Mouth-to-Mouth or Basic Cardiac Life Support or Life Support, B a si c C a rdiac); \# 2 all=(Hypothermia, Induced or Therapeutic Hypothermia or Hypothermia, Therapeutic or Targeted Temperature Management or Targeted Temperature Managements or Induced Hypothermia or Moderate Hypothermia, Induced or Induced Moderate Hypothermia or Induced Moderate Hypothermias or Moderate Hypothermias, Induced or Mild Hypothermia, Induced or Induced Mild Hypothermia or Induced Mild Hypothermias or Mild Hypothermias, Induced); \#3 all=(Heart Arrest or Arrest, Heart or Cardiac Arrest or Arrest, Cardiac or Asystole or Asystoles or Cardiopulmonary Arrest or Arrest, Cardiopulmonary or ((pulseless* or nonperfus*) and (ventricular tachycardia* or ventricular tachyarrhythmia)) or ventricular fibrillation* or Pulseless electrical activity or PEA); \#4 \#1 AND \#2 AND \#3.

Participant or population: Patents with cardiac arrest and received targeted temperature management (TTM) or not.

Intervention: The different time of collapse to return of spontaneous circulartion (<20min+TTM, <20min, 20-39min+TTM, 20-39min, 40-59min+TTM, 40-59min, $\geq 60 \mathrm{~min}+\mathrm{TTM}, \geq 60 \mathrm{~min}$ ).

\section{Comparator: Same as intervention.}

Study designs to be included: All type eligible study.

Eligibility criteria: Trials that met the following criteria were included: a) patients in the trial underwent CA whether IHCA or OHCA; b) valid data of 30-day or at discharge survival neurologic outcome could be extracted; c) the time of collapse to ROSC could be extracted and we could divide into groups based on the time of collapse to ROSC; d) if patients receive TTM the temperature should range from $32^{\circ} \mathrm{C}$ to $36^{\circ} \mathrm{C}$.
Information sources: We performed this network meta-analysis by searching four online databases (PubMed, Embase, CENTRAL, and Web of Science) up to date month 2021. Moreover, references of relevant meta-analyses, letters, editorials, reviews, and eligible trials were also screened.

Main outcome(s): 30 -day or at discharge survival or good neurologic outcome.

Additional outcome(s): None.

Data management: A Bayesian random effects model for analyzing multiple treatment groups was performed to compare 30-day or at discharge survival and good neurologic outcome among different groups. We used Bayesian extension of the hierarchical random effects model proposed by Lumley for networks of multi-arm trials. A Bayesian network meta-analysis can make direct or indirect comparisons whether there was a direct comparison between the two groups. Pooled data was analyzed via STATA/MP 16.0 (Stata Corp LP, College Station, Texas, USA). Markov chain Monte Carlo samplers were run in Stata, and 4 chains was running with different starting values. Vague, noninformative prior distributions with very small precision were given. A burn-in phase of 20,000 iterations was used to ensure convergence. The convergence was checked by running 4 chains at different starting values using the Gelman-Rubin methods, which were stable in all instances. For inference, 50,000 iterations were used. A Fixed effect model was use in traditional frequentist meta-analysis to analyze pooled odds ratios (OR). The traditional frequentist meta-analysis was performed with the use of STATA. Pairwise OR were estimated from the median of the posterior distribution with credible intervals (CI) taken from the $2.5 \%$ and $97.5 \%$ percentiles. The result was considered significantly different when $\mathrm{Cl}$ did not include 1. Markov chain Monte Carlo (MCMC) modeling was used to calculate the relative ranking probability of each intervention. "Rankograms" along with surface under the cumulative ranking curve 
(SUCRA) were provided to compare hierarchy of efficacy and safety of the interventions. The SUCRA is a numeric presentation of the overall ranking and demonstrates a single number associated with each treatment. The SUCRA values range from 0 to $100 \%$. The higher the SUCRA value, and the closer to $100 \%$, the higher the likelihood that a group is in the top rank or highly effective; the closer to 0 the SUCRA value, the more likely that a group is in the bottom rank or ineffective. Moreover, funnel plot was performed to assess the publication bias (Supplementary Figure 4 and 8). If the funnel plot was not evenly distributed, we consider publication bias in these trials. Node-splitting models were constructed to assess the level of inconsistency between the estimates from direct and indirect evidence.

Quality assessment / Risk of bias analysis: Cochrane Handbook version 5.1.0 was used to assess the quality of RCT and risk of bias (Supplementary Figure 9 and 10). Meanwhile, the Newcastle-Ottawa Assessment Scale (NOS) for control study or cohort study was used to assess the quality and risk of bias of retrospective or cohort study respectively (Supplementary Table 5 and 6). We considered that the score of NOS more than seven is high quality.

Strategy of data synthesis: Published data from the included trials were pooled used in this network meta-analysis. We performed a standard method to extract demographics and the characteristics of the included trials. Finally, 30-day or at discharge survival or good neurologic outcome, as the pre-specific endpoint, was collected from the included trials.

Subgroup analysis: None.

Sensitivity analysis: None.

Language: None limitation.

Country(ies) involved: China.

Other relevant information: None.
Keywords: cardiac arrest; targeted temperature management; time of return of spontaneous circulation; survival; good neurologic outcome.

Contributions of each author:

Author 1 - Jingwei duan.

Author 2 - Jie Yu.

Author 3 - Qiangrong Zhai.

Author 4 - Qingbian Ma. 